\title{
News-vendor game-based resource allocation scheme for next-generation C-RAN systems
}

\author{
Sungwook Kim
}

\begin{abstract}
Recently, Cloud Radio Access Network (C-RAN) has been emerging as a cost-effective solution supporting huge volumes of mobile traffic in the big data era. C-RAN provides infrastructure layer services to mobile users by managing virtualized infrastructure resources. To exploit next-generation C-RAN operations, a main challenging issue is how to properly control system resources. In this study, we propose a novel resource management scheme for C-RAN systems. By employing the news-vendor game model, we investigate a resource allocation problem with bargaining solutions. In dynamic C-RAN environments, our game-based resource management approach can practically adapt current system conditions while maximizing the expected payoff. The main contribution of our study lies in the fact that we shed a new light on adaptive resource allocation policies for virtualized cloud environments. Simulation results demonstrate that our proposed scheme can obtain a considerably better system performance than other existing schemes.
\end{abstract}

Keywords: News-vendor game, Dynamic resource allocation, Bargaining solutions, Cloud Radio Access Networks, Quality of service, Virtualization

\section{Introduction}

Modern computation and communication systems operate in a new and dynamic world, characterized by continual changes in the environment and performance requirements that must be satisfied. Dynamic system changes occur without warning and in an unpredictable manner, which are outside the control of traditional operation approaches [1]. At the same time, popularity of mobile devices and related applications in various fields are increasing significantly in everyday life. Furthermore, applications become more and more complex, quality of service (QoS) sensitive and computation intensive to perform on mobile system. Therefore, new solution concepts need to be developed that manage the computation and communication systems in a dynamically adaptive manner while continuously ensuring different application services [1,2].

Cloud Radio Access Network (C-RAN) is a new system architecture for the future mobile network

Correspondence: swkim01@sogang.ac.kr

Department of Computer Science, Sogang University, Shinsu-dong 1, Mapo-ku, Seoul 121-742, South Korea

infrastructure. It is a centralized, cloud computing-based new radio access network to support future wireless communication standards. C-RAN can be implemented based on the concept of virtualization. Usually, virtualization is an enabling technology that allows sharing of the same physical machine by multiple end-user applications with QoS guarantees. Therefore, it helps to reduce costs while improving a higher utilization of the physical resources $[1,3]$. In the C-RAN system, baseband processing unit of traditional physical machines are pooled and moved into a centralized location. Through virtualization, the computing resources in the baseband unit pool can be dynamically shared among all applications in the C-RAN system while allowing a significant improvement in computing resource utilization and power efficiency [4]

No one may deny the advantages of C-RAN services. However, there are some problems that need to be addressed. Most of all, next-generation C-RAN systems should take into account QoS guarantees while maximizing resource efficiency. However, because of the scarcity of system resource, it is difficult to satisfy 
simultaneously these conflicting requirements. For this reason, the most critical issue for the next-generation $\mathrm{C}$ RAN system is to develop effective resource allocation algorithms [5]. But despite flexibility and great potential applicability, resource allocation problem in C-RAN has received scarce attention as of today.

To design a resource allocation algorithm in C-RAN systems, it is necessary to study a strategic decisionmaking process. Under widely dynamic C-RAN conditions, system agents can be assumed as intelligent rational decision-makers, and they select a best-response strategy to maximize their expected utility with other agents. This situation is well-suited for the game theory. Game theory is a field of applied mathematics that provides an effective tool to model interactions among independent decisionmakers. It can describe the reactions of one set of decision-makers to another and analyze the situations in terms of conflict and cooperation. Thus, game theory can be a major paradigm for modeling resource allocation problems that feature complex interactive relations [6].

News-vendor game $[7,8]$ is a mathematical game model in operations management and applied economics used to determine optimal inventory levels. Typically, it is characterized by fixed prices and uncertain demand for a perishable product. Therefore, this model can represent a situation faced by a newspaper vendor who must decide how many copies of the day's paper to stock in the face of uncertain demand and knowing that unsold copies will be worthless at the end of the day. The original concept of news-vendor game appeared to date from 1888 where F. Edgeworth used the central limit theorem to determine the optimal cash reserves to satisfy random withdrawals from depositors. The modern formulation dates from the 1951 paper in Econometrica by K. Arrow, T. Harris, and J. Marshak [9].

Motivated by the aforementioned discussion, we design a new game-theoretic resource allocation scheme for C-RAN systems. The main goal of our proposed scheme is to maximize resource efficiency while providing QoS guarantees. In dynamically changing C-RAN environments, our game process is divided two stages; the competitive stage and the bargaining stage. At the competitive stage, system resource is allocated in a noncooperative game manner. Therefore, cloud server controls dynamically the total service request by adjusting the price. When the total service request is larger than the system capacity with the maximum price, system resource cannot be distributed effectively in a noncooperative manner. To effectively handle this case, we adopt a bargaining-based approach. At the bargaining stage, we re-distribute the system resource on the basis of combined bargaining solution.

According to our two-stage coordinative game paradigm, we can find an effective solution that can retain several desirable features for real-world C-RAN operations. In this study, the main novelty lies in the fact that we develop a novel resource allocation scheme based on the news-vendor game model, and a fair-efficient solution is obtained through the two-stage game paradigm, which is implemented by employing a cooperative and competitive decision process. To capture the feature of C-RAN system dynamics, it is a hot research issue to design a new game paradigm. However, to the best of our knowledge, relatively little research has been done on this issue over the years.

\subsection{Related work}

The area of numerical methods or algorithms for efficient C-RAN resource sharing problems has been extensively studied and has received considerable attention in recent years [1, 5, 10-14]. The Joint Cloud Computing and Network (JCCN) scheme [10] was proposed to jointly study dynamic clouds and wireless network operations so as to improve end-to-end performance in the mobile cloud computing environment. This scheme considered not only the spectrum efficiency in wireless networks but also the pricing information in the cloud, based on which power allocation and interference management in wireless networks were performed. The JCCN scheme formulated the problems of cloud media service price decision, resource allocation and the interference management in the mobile cloud computing environment as a three-level Stackelberg game [10].

Ali et al. developed the Cloud Resource Bartering $(C R B)$ model for sharing user's computational resources through a social network [12]. The CRB model allowed users of online social network to share their cloud resources without money changing hands. This scheme linked a social network with the computational cloud to create a social cloud so that users can share their part of the cloud with their social community [12].

The Social Compute Cloud (SCC) scheme [13] was developed for the social cloud interaction system. This scheme has presented a social compute cloud platform that enabled the sharing of infrastructure resources between friends via digitally encoded social relationships. To construct a social compute cloud, the SCC scheme accessed users' social networks, allowed users to elicit sharing preferences, and utilized matching algorithms to enable preference-based socially aware resource allocation.

The Cloud Provider's Resource Sharing (CPRS) scheme [11] was developed to study the cooperative behavior of multiple cloud providers. In the CPRS scheme, a hierarchical cooperative game model was designed; it was composed of two interrelated cooperative games to analyze the decisions of cloud providers to support internal users and to offer service to public cloud users. In 
the lower level, the CPRS scheme implemented a stochastic linear programming game model to study the resource and revenue sharing for a given coalition of cloud providers. In the upper level, the CPRS scheme formulated the coalitional game for which the cloud providers can form the groups of cooperation to share resource and revenue. Finally, the analytical model based on Markov chain was used to obtain stable coalitional structure [11].

The Reputation-based Social Cloud (RSC) scheme [14] added the concept of reputations as part of the utility. This scheme described the architecture and interaction between two rational parties in the social cloud, where two parties received their opponent's trust or reputation from the social cloud. In the RSC scheme, the reputation was affected by the interactions with other parties in the social network. As mentioned above, numerous studies have shown how social networks create social influences on people's choices across time and space.

The Joint Resource Allocation (JRA) scheme [15] presented a resource allocation algorithm for deviceto-device communications underlaying uplink MIMO cellular networks. The main aim of the JRA scheme was to solve the sum-rate maximization problem. Based on the non-cooperative resource allocation game model, a distributed resource allocation method was devised. It was proved that the algorithm converged to the feasible pure strategy Nash equilibrium under specific conditions. Furthermore, a sum-rate reinforcement approach was also proposed to address the case when the proposed distributed algorithm did not converge [15].

The Discrete Power Control (DPC) scheme [16] investigated the joint relay selection and discrete power control problem for cognitive relay networks. The problem was formulated as a non-cooperative potential game which possessed at least one pure strategy Nash equilibrium. Under some mild conditions, a centralized algorithm and a decentralized learning algorithm had been proposed to obtain a locally optimal solution. The $D P C$ scheme can guarantee the feasibility of a pure strategy Nash equilibrium without advance knowledge of infeasible strategy profiles [16].

The Hierarchical Cloud Resource Management (HCRM) scheme in [1] was proposed to devise resource allocation policies for virtualized cloud environments that must satisfy performance and availability guarantees. In particular, the HCRM scheme managed the transactional service applications of its customers to satisfy response time and availability guarantees while minimizing energy costs in very large cloud service centers. Based on a hierarchical framework, this scheme can provide availability guarantees for the running applications. Finally, the effectiveness of the HCRM scheme was assessed by considering realistic workloads [1].
The Multi-dimensional Dynamic Resource Management (MDRM) scheme in [5] was a model for mobile application profiles, wireless interfaces, and cloud resources. Based on the dynamic constraint programming method, the MDRM scheme took network parameters, mobile devices, and application constraints as input to optimally select the network resources and application QoS profiles. Under different environmental variables, this scheme could be dynamically adaptive to environmental parameter variation. At the end, numerical results showed that the MDRM scheme saved the mobile battery life and guaranteed both QoS and cost simultaneously. All the earlier work has attracted a lot of attention and introduced unique challenges. In this study, we compared the performance of the proposed scheme with that of the HCRM scheme [1] and the MDRM scheme [5] to confirm the superiority of our approach.

The remainder of this paper is organized as follows: In Section 2, we formulate the system model and describe concretely our resource allocation algorithm. In Section 3 , the simulation scenario is presented, where the traffic model is described and a numerical result analysis is presented. The paper concludes with a discussion of the results in Section 4.

\section{Proposed C-RAN resource allocation algorithm}

In this section, the proposed resource allocation scheme is explained in detail. Based on the news-vendor game model, our proposed scheme can provide a globally desirable system performance while ensuring the efficiency of C-RAN resource.

\subsection{News-vendor game models at the competitive stage} C-RAN has come to the key architecture concept for future 5G networks and beyond. In C-RAN systems, virtualized baseband units pool $(V B P)$ arbitrates multiple cloud providers $(C P s)$ and collects available resources. To ensure the optimal usage of cloud resource, the VBP adaptively distributes this resource to multiple base stations $(B S s)$. In wireless networks, each $B S$ covers a small area and communicates with the mobile users $(M U s)$ through wireless links. Therefore, BSs must provide the managed connectivity and offer flexibility in real-time demands. To improve C-RAN system efficiency, $C P s$ offer their available resources to $B S s$ through the $V B P$, and $B S s$ provide services to $M U s$ based on their obtained resources. Without loss of generality, the $V B P$ can be assumed as a resource broker between $B S s$ and $C P S$ for cloud services [17].

In this section, we introduce our news-vendor game model $(\mathbb{G})$ for C-RAN systems. $\mathbb{G}$ is a tuple $\left(V, \mathbb{N},\left(S_{i}\right)_{i \in \mathbb{N}},\left(U_{i}\right)_{i \in N}, T\right)$ at each time period $t$ of gameplay. 
- $V$ is the total amount of available cloud resource in the $V B P$.

- $\mathbb{N}$ is the finite set of players $\mathbb{N}=\left\{b_{0}, b_{1}, \ldots, b_{n}\right\}$ where $b_{0}$ is the $V B P$ and $b_{i, 1 \leq i \leq n}$ represents the $i$ th $B S$.

- $S_{i}$ is the set of strategies with the player $i$. If the player $i$ is the $V B P$, i.e., $i=0$, a strategy set can be defined as resource prices. If the player $i$ is a $B S$, i.e., $1 \leq i \leq n$, the strategy set is defined as the amount of requested resource.

- The $U_{i}$ is the payoff received by the player $i$. Traditionally, the payoff is determined as the obtained outcome minus the cost to obtain that outcome. For simplicity, the outcome is represented in a general form of $\log$ function.

- The $T$ is a time period. The $\mathbb{G}$ is repeated $t \in T<\infty$ time periods with competitive and cooperative manner.

To understand the behavior of self-regarding system agents, game models have some attractive features. As a kind of game model, news-vendor game was initially developed for the classical, single-period newsboy problem [18]. In this study, the traditional news-vendor game is extended as a two-stage repeated game. Initially, the $V B P$ dynamically adjusts the price of resource unit, and $B S s$ request cloud resources to maximize their payoffs. In this stage, resource allocation procedure is formulated as a non-cooperative game model. If service requests from $B S s$ are more than the capacity of the $V B P$, the cloud resource is re-distributed adaptively according to our combined bargaining solution. In this stage, resource allocation procedure is formulated as a bargaining game approach. By a sophisticated combination of these two different game approaches, we attempt to approximate a well-balanced system performance among conflicting requirements.

At the competitive stage, the strategy set for the $V B P$ $\left(S_{0}\right)$, i.e., available price levels for a resource unit, is assumed as below.

$$
\boldsymbol{S}_{0}=\left\{p^{t} \mid p^{t} \in\left[p_{\min }, p_{\max }\right]\right\}
$$

where $p^{t}$ is the price at time $t$. The $p_{\min }, p$ max are the pre-defined minimum and maximum price levels, respectively. From the viewpoint of $V B P$, the $p_{\max }$ is good to maximize its profit. From the viewpoint of $B S s, p_{\text {min }}$ is good to maximize their payoff. The actual price at time $t\left(p^{t}\right)$ is dynamically decided according to the current system conditions. In our model, the $p^{t}$ is determined as the weighted sum of $p_{\min }$ and $p_{\max }$.

$$
p^{t}=\omega \times p_{\max }+(1-\omega) \times p_{\text {min }}
$$

where $\omega$ is a weighted factor for the both prices. Under diverse system environments, the value of $\omega$ should be modified dynamically. In the proposed scheme, Rubinstein-Stahl model is adopted to adjust the $\omega$ value. Rubinstein-Stahl model was proposed as a solution to the problem when two players were negotiating the division of the benefit [19]. Therefore, players negotiated with each other by proposing offers alternately. After several rounds of offer and countoffers, players finally come to an agreement. In Rubinstein-Stahl model, there exists a unique solution for this negotiation process [19]. We assume that the Rubinstein-Stahl model's equilibrium point is obtained through negotiation between the $V B P$ stance and $B S s^{\prime}$ stance.

In our Rubinstein-Stahl model, the $V B P$ is assumed as a supplier, and all BSs are assumed as a single customer. Two players, i.e., supplier and customer, have their own bargaining power $(\delta)$. The division proportion of the benefits can be obtained according to the negotiation power, which can be computed at each player individually. A more negotiation power player benefits more from the negotiation process. Players negotiate with each other by proposing offers alternately. After several rounds of negotiation, they finally reach an agreement as following [20, 21].

$$
\left(x_{s}^{*}, x_{c}^{*}\right)=\left\{\begin{array}{l}
\left(\frac{1-\delta_{c}}{1-\delta_{s} \times \delta_{c}}, \frac{\delta_{c} \times\left(1-\delta_{s}\right)}{1-\delta_{s} \times \delta_{c}}\right) \text { if supplier offers first } \\
\left(\frac{\delta_{s} \times\left(1-\delta_{c}\right)}{1-\delta_{s} \times \delta_{c}}, \frac{1-\delta_{s}}{1-\delta_{s} \times \delta_{c}}\right) \text { if customer offers first }
\end{array}\right.
$$

$$
\begin{aligned}
\text { s.t., }\left(x_{s}^{*}, x_{c}^{*}\right) \in \mathbf{R}^{2} & : x_{s}^{*}+x_{c}^{*} \\
& =1, x_{s}^{*} \geq 0, x_{c}^{*} \geq 0 \text { and } 0 \leq \delta_{s}, \delta_{c} \leq 1
\end{aligned}
$$

where $x_{s}^{*}$ and $x_{c}^{*}$ are final dividends for supplier and customer, respectively. $\delta_{s}$ and $\delta_{c}$ be the supplier and consumer patience factor. Lower $\delta$ (or higher $\delta$ ) value means lower patience (or more patience). In the Rubinstein-Stahl negotiation model, the patience factor strongly affects the negotiation process; the more patience has, the more payoff attains. From a common-sense standpoint, consumers should know the current price as early as possible for the effective service continuity. Under this situation, they lack patience in bargaining. For this reason, we represent the consumer's patience as a monotonous time-decreasing function. According to the inverse effect of reciprocal relationship, the supplier's patience is defined vice versa. Therefore, the consumer's patience $\left(\delta_{c}^{t}\right)$ and supplier's patience $\left(\delta_{s}^{t}\right)$ at th round of negotiation process are defined as follows $[19,22]$. 


$$
\begin{aligned}
\delta_{c}^{t} & =1-\left(\frac{e^{\xi^{t}}-e^{-\xi^{t}}}{e^{\xi^{t}}+e^{-\xi^{t}}}\right) \quad \text { and } \quad \delta_{s}^{t} \\
& =\left(\frac{e^{\xi^{t}}-e^{-\xi^{t}}}{e^{\xi^{t}}+e^{-\xi^{t}}}\right) \\
\text { s.t., } & \frac{d \delta_{c}^{t}}{d t}<0, \quad \frac{d \delta_{s}^{t}}{d t}>0, \quad \delta_{c}^{0}, \delta_{s}^{\infty}=1 \text { and } \delta_{c}^{\infty}, \delta_{s}^{0} \\
& =0
\end{aligned}
$$

where $\xi^{t}$ is the patience coefficient at $t$ th round. For the ideal C-RAN system management, we dynamically adjust the $\xi^{t}$ value. When the requested service increases (or decreases), the price ( $p$ ) should increase (or decrease) to maximize the resource efficiency. To implement this mechanism, the value of $\xi^{t}$ is defined as the ratio of the current cloud workload to the total system capacity.

$$
\xi^{t}=1-\left(\frac{T_{Q}-C_{Q}}{T_{Q}}\right), \quad \text { s.t., } 0<\xi^{t}<1
$$

where $T_{Q}$ and $C_{Q}$ are the total cloud capacity and the current cloud workload, respectively. If the gap between $T_{Q}$ and $C_{Q}$ is larger, the $\xi^{t}$ decreases using (5) and $\delta_{c}$ (or $\delta_{s}$ ) increases (or decreases), simultaneously. Therefore, according to the $\xi^{t}$ value, the values of $\delta_{c}$ and $\delta_{s}$ are adjusted adaptively. On the basis of obtained $\delta_{c}$ and $\delta_{s}$ values, we can get the weighted factor $(\omega)$. In a realistic negotiation scenario, a supplier offers the price first. Therefore, the values of $\omega$ and (1- $\omega)$ are obtained according to (3).

$$
\begin{aligned}
\omega & =\frac{1-\delta_{c}}{1-\delta_{s} \times \delta_{c}} \quad \text { and } \quad(1-\omega) \\
& =\frac{\delta_{c} \times\left(1-\delta_{s}\right)}{1-\delta_{s} \times \delta_{c}}
\end{aligned}
$$

Finally, the price $(p)$ in the competitive game stage is obtained based on Eq. (2). When the $p$ is high, cloud service requests are reduced with unsatisfactory payoffs, and vice versa. Therefore, at the competitive stage, the $V B P$ can control dynamically the total service request by adjusting the price according to (2), (3), (4), (5), and (6).

\subsection{News-vendor game models at the cooperative stage}

When the current cloud workload is controllable through the proposed price strategy, our news-vendor game can be operated only in the competitive game stage. However, in an overloaded situation, i.e., the cloud resource is not sufficient to support all service requests, our cooperative game stage is started. In recent years, cooperative approaches derived from game theory have been widely used for efficient resource allocation problems. The most popular approaches are the Nash bargaining solution (NBS) and the Kalai-Smorodinsky bargaining solution (KSBS) $[6,23]$. Because of their appealing properties, the basic concept of NBS and KSBS has become an interesting research topic in economics, political science, sociology, psychology, biology, and so on [6].

Based on the traditional game theory, the Nash bargaining solution can be formulated as follows [6].

$$
\prod_{i}\left(u_{i}^{*}-d_{i}\right)=\max _{u_{i} \in \mathbb{S}} \prod_{i}\left(u_{i}-d_{i}\right), \text { where } u_{i}^{*} \in \mathbb{S} \text { and } d_{i} \in \boldsymbol{d}
$$

where $\mathbb{S}=\left\{\left(u_{1}, \ldots u_{n}\right)\right\} \subset \mathbb{R}^{\mathrm{n}}$ is a jointly feasible utility solution set, and a disagreement point $(\boldsymbol{d})$ is an action vector $\boldsymbol{d}=\left(d_{1}, \ldots d_{n}\right) \in \mathbb{S}$ that is expected to be the result if players cannot reach an agreement; it represents payoffs when the cooperative game fails and the resource allocation cannot be made (i.e., zero in the system). Therefore, the NBS is obtained by maximizing the product of the utilities $(u)$. In this study, $u_{i}$ is defined as the throughput of $i$ th $B S$. In the game theory terminology, an outcome vector $\left\langle u_{1}^{*}, u_{2}^{*},,, u_{n}^{*}\right\rangle$ is a unique and fair-efficient solution, called the NBS that fulfills the Nash axioms [6].

(1)Individual rationality: NBS should be better off than the disagreement point. Therefore, no player is worse off than if the agreement fails. Formally, $u_{i}^{*} \geq d_{i}$ for all player $i$.

(2)Feasibility: NBS is reasonable under the circumstances. That is, $U^{*} \in \mathbb{S}$.

(3) Pareto optimality: NBS gives the maximum payoff to the players. Therefore, if there exists a solution $U^{*}$ $=\left(u_{1}^{*} . . u_{i}^{*} . . u_{n}^{*}\right)$, it shall be Pareto optimal.

(4)Invariance with respect to utility transformations: A utility function specifies a player's preferences. Therefore, different utility functions can be used to model the same preferences. However, the final outcome should not depend on which of these equivalent utility representations is used. In other words, for any linear scale transformation of the function $\psi, \psi(F(\mathbb{S}, \mathrm{d}))=F(\psi(\mathbb{S}), \psi(\mathrm{d}))$. This axiom is also called independence of linear transformations or scale covariance.

(5)Independence of irrelevant alternatives: The solution should be independent of irrelevant alternatives. In other words, a reasonable outcome will be feasible after some payoff sets have been removed. If $U^{*}$ is a bargaining solution for a bargaining set $\mathbb{S}$ then for any subset $\mathbb{S}^{\prime}$ of $\mathbb{S}$ containing $U^{*}, U^{*}$ continues to be a bargaining solution. Formally, if $U^{*} \in \mathbb{S}^{\prime} \subset \mathbb{S}$ and $U^{*}=F(\mathbb{S}, \mathrm{d})$, then $U^{*}=F\left(\mathbb{S}^{\prime}, \mathrm{d}\right)$.

(6) Symmetry: Symmetry means that if the players' utilities are exactly the same, they should get symmetric payoffs, i.e., equal payoffs. Therefore, 
payoff should not discriminate between the identities of the players but only depend on utility functions. For example, if $\mathbb{S}$ is invariant under all exchanges of users, $F_{i}(\mathbb{S}, \mathrm{d})=F_{j}(\mathbb{S}, \mathrm{d})$ for all possible players $i$ and $j$.

Even though the NBS can provide a unique and fair Pareto optimal solution, Nash axioms do not always characterize the situations we encounter in reality. In particular, the independence of irrelevant alternatives has been the source of considerable contention. When a feasible solution set is modified, NBS is unconcerned about a relative fairness. Therefore, the dilemma is an insensitivity to utility translations. In some cases, the outcome of the bargaining process may be the result of reciprocal equality. Therefore, during the 1950-1980s, extensive research had been done to replace the axiom, independence of irrelevant alternatives [24].

KSBS is an alternative approach to the bargaining problem proposed by Kalai and Smorodinsky [6, 23]. While Nash's solution requires the solution to be independent when irrelevant alternatives are modified, the KSBS relaxed this condition. Therefore, Kalai and Smorodinsky replaced the axiom of independence of irrelevant alternatives by individual monotonicity. Under individual monotonicity condition, if the feasible set is changed in favor of one of the players, this player should not end up losing because of this change [24]. More formally, individual monotonicity axiom is defined as $[6,25]$.

(7) Individual monotonicity: A bargaining situation $(\mathbb{W}, d)$ is better than $(\mathbb{S}, d)$ if and only if sup $\left\{u_{i}:\left\{\left(u_{1},,, u_{n}\right)\right\} \in \mathbb{W}\right\} \geq \sup \left\{u_{i}:\left\{\left(u_{1},,, u_{n}\right)\right\} \in \mathbb{S}\right\}$ where $1 \leq i \leq n$. A solution function $F$ is individually monotonic for a player if whenever $(\mathbb{W}, d)$ is better than $(\mathbb{S}, \mathrm{d})$, then $F(\mathbb{W}, \mathrm{d})>F(\mathbb{S}, \mathrm{d}) . F$ is individually monotonic if the same property holds for all players.

KSBS is a unique solution satisfying the axioms (1), (2), (3), (4), (6), and (7). Mathematically, it is defined as $[6,24,25]$.

$$
\begin{aligned}
\frac{\sup \left\{u_{1}\right\}-d_{1}}{I_{1}^{*}-d_{1}} & =\ldots=\frac{\sup \left\{u_{i}\right\}-d_{i}}{I_{i}^{*}-d_{i}}=\ldots \\
& =\frac{\sup \left\{u_{n}\right\}-d_{n}}{I_{n}^{*}-d_{n}} \\
\text { s.t., } \sup \left\{u_{i}\right\} & =\sup \left\{u_{i}:\left\{\left(u_{1},,, u_{n}\right)\right\} \in \mathbb{W}\right\}, I_{i}^{*} \\
& =\max \left\{u_{i}: u_{i} \in \mathbb{S}\right\} \text { and } 1 \leq i \leq n
\end{aligned}
$$

where $I_{i}^{*}$ is the ideal point of player $i$. Therefore, players choose the best outcome subject to the condition that their proportional part of the excess over the disagreement is relative to the proportion of the excess of their ideal gains $[6,24,25]$. Simply, we can think that the KSBS is the intersection point between the Pareto boundary and the line connecting the disagreement to the ideal gains. To help understand the full concept of NBS and KSBS, simple examples of two-player case are graphically depicted in Fig. 1.

In this study, we develop a new bargaining solution by combining the axioms of independence of irrelevant alternatives and individual monotonicity. To implement our solution, the main issues are how to trade-off between different principles, which can be tackled by cooperative games with transferable utility. The combined bargaining solution $\left(u^{\alpha}\right)$ of the NBS and KSBS is obtained as a weighted average:

$$
\begin{aligned}
& u_{i}^{\alpha}=\alpha \times u_{i}^{\mathrm{NBS}}+(1-\alpha) \times u_{i}^{\mathrm{KSBS}} \\
& \text { s.t., } \quad u_{i}^{\alpha} \in\left\{u_{1}^{\alpha} . . u_{i}^{\alpha} . . u_{n}^{\alpha}\right\} \text { and } 1 \leq i \leq n
\end{aligned}
$$

where $\alpha$ is a control parameter to relatively emphasize the principle of Independence of irrelevant alternatives or Individual monotonicity. The major feature of individual monotonicity is that increasing the bargaining set size in a direction favorable to a specific player always benefits that player. Therefore, when the bargaining set size of each player is huge different, this feature can keep the relative fairness among players.

In this study, we adaptively adjust the $\alpha$ value in an online decision manner. In dynamic C-RAN environments, a fixed value of $\alpha$ cannot effectively adapt to the changing conditions. When the normalized difference of the bargaining set size is high, we should strongly depend on the axiom of individual monotonicity. In this case, a lower value of $\alpha$ is more suitable. When the normalized difference of the bargaining set size is nearly the same, we can put more emphasis on the axiom of independence of irrelevant alternatives. In this case, a higher value of $\alpha$ is more desirable. Based on this consideration, the value of $\alpha$ is dynamically adjusted according to the current ratio of bargaining set difference.

$$
=\frac{\min _{i, j \in \mathrm{N}}\left(\left|I_{i}^{*}-I_{j}^{*}\right|\right)}{\max _{i, j \in \mathrm{N}}\left(\left|I_{i}^{*}-I_{j}^{*}\right|\right)} \quad \text {, s.t., } 1 \leq i, j \leq n
$$

With the dynamic adaptation of $\alpha$ value, the proposed scheme can be more responsive to current C-RAN conditions. Finally, the set of resource allocation for BSs at time $t$, denoted by $\mathbb{R}$, is calculated as 




(a) NBS

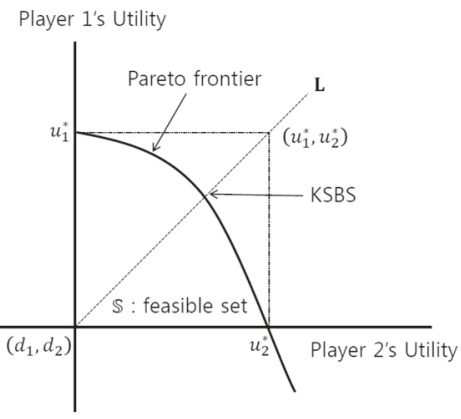

(b) KSBS

Fig. 1 Simple examples of NBS (a) and KSBS (b) for the two-player case

$$
\mathbb{R}=\left\langle\mathscr{R}_{i} \mid\left\{\mathscr{R}_{1} \ldots \mathscr{R}_{i} \ldots \mathscr{R}_{n}\right\}\right\rangle \stackrel{\text { def }}{=} \mathscr{R}_{i}=\left(\frac{u_{i}^{\alpha}}{\sum_{i=1}^{n} u_{i}^{\alpha}}\right) \times \mathfrak{R}^{t}
$$

where $\Re^{t}$ is the available cloud resource at time $t$. The solution set $\mathbb{R}$ is a possible outcome of our combined bargaining process; $\mathbb{R}$ is adaptively obtained in the cooperative trade-off area.

\subsection{The main steps of proposed resource allocation algorithm}

Presently, C-RAN draws significant attention in the information technology community as it provides ubiquitous on-demand access to a shared pool of configurable computing resources with minimum management effort. For effective C-RAN operations, the cloud resource must be distributed dynamically for BSs. However, it is questionable whether existing resource allocation solutions are adequate in dynamically changing C-RAN environments. In this study, we have investigated the benefits and challenges of resource allocation algorithm in C-RAN architecture. The major objective of this study is to design a novel for C-RAN resource allocation scheme based on the news-vendor game. In the proposed scheme, both non-cooperative and cooperative game models have been applied to the cloud resource allocation process. Our two-stage game approach is an effective way to control the C-RAN cloud resource. At the competitive stage, the resource is allocated dynamically in a non-cooperative game manner. Through the control of the resource price, the $V B P$ and $B S s$ attempt to maximize their payoffs. At the bargaining stage, the cloud resource is distributed to BSs according to our combined bargaining solution. The proposed two-stage approach suggests that a judicious mixture of collaboration and competition is advantageous in dynamic CRAN environments. The main steps of the proposed resource allocation algorithm are given next.
Step 1: At the initial time, the price $(p)$ is set to the initial value and $B S s$ request their cloud service to maximize their payoffs in a non-cooperative game approach.

Step 2: At each game period, the $p$ is decided according to the Rubinstein-Stahl model. In the basis of (2), (3), (4), (5), and (6), the $p$ is dynamically adjusted by taking into account the current cloud workload ( $\xi$ ).

Step 3: After the $p$ decision, $\delta, \omega$, and $\xi$ values are modified periodically using (4),(5), and (6).

Step 4: When the cloud services are congested at the competitive stage, it is impossible to control the resource allocation through price control strategy. At this time, the bargaining stage is started.

Step 5: At each game period, NBS and KSBS are obtained using (7) and (8). At the same time, the control parameter $\alpha$ is adjusted dynamically using (10).

Step 6: The combined bargaining solution $\left(u_{i, 1 \leq i \leq n}^{\alpha}\right)$ of the NBS and KSBS are obtained based on (9), and the set of resource allocation for $B S s(\mathbb{R})$ is finally calculated according to (11).

Step 7: Under widely diverse C-RAN environments, the $V B P$ and $B S s$ are self-monitoring constantly for the next news-vendor game process; proceed to Step 2.

\section{Performance evaluation}

In this section, the effectiveness of the proposed scheme is validated through simulation. Using a simulation model, the performance of the proposed scheme is compared with two existing schemes: the HCRM scheme [1] and the MDRM scheme [5]. The assumptions implemented in simulation model are as follows.

- The simulated model is assumed as a C-RAN system with one $V B P$ and five $S B S s$.

- The process for new application service requests in each BS is Poisson with rate $\sigma$ (applications/s), and the range of offered load was varied from 0 to 3.0. 
- The total cloud resource $(V)$ is CPU computation capacity, and its capacity is $5 \mathrm{GHz}$.

- System performance measures obtained on the basis of 50 simulation runs are plotted as a function of the offered application service load.

- Each application service has its own application type and requires different resources requirements. They are generated with equal probability.

- The durations of services are exponentially distributed.

- The C-RAN system performance is estimated in terms of the normalized payoff, C-RAN resource efficiency, system fairness, and QoS satisfaction probability.

In order to emulate a real C-RAN system environment and for a fair comparison, application types, characteristics and system parameters are carefully selected for a realistic simulation scenario. Table 1 shows the application types and system parameters used in our simulation.

As mentioned earlier, the HCRM scheme [1] and the $M D R M$ scheme [5] have been recently published and introduced unique challenges to efficiently solve the resource allocation problem in C-RAN systems. However, they are successful only in certain circumstances. Compared to these schemes, we can confirm the superiority of our proposed two-stage game approach. Through Figs. 2, 3, 4, and 5, the $x$-axis (a horizontal line) marks the service load intensities, which is varied from 0 to 3.0. Based on each rate of offered service load, performance criteria are evaluated as a normalized value; $y$-axis (a vertical line) represents the normalized value for each performance criteria.

Figure 2 shows the performance comparison in terms of the normalized payoff of each scheme. It is measured as a normalized utility sum of all BSs. To maximize the C-RAN system performance, it is an important performance metric. Under various application service requests, our two-stage game-based approach effectively controls resources. From the simulation results, the main observation is that our proposed scheme can effectively allocate cloud resource and lead to a higher payoff than other existing methods. When designing an effective resource allocation algorithm for C-RAN system, it is a highly desirable property.

Figure 3 presents the performance comparison in terms of the resource efficiency in the C-RAN system. In general, resource efficiency is the ratio of actively used resource amount over the total resource amount. A key observation from the results shown in Fig. 3 is that all the schemes have similar trends. This trend implies that under higher service requests, a better resource efficiency is obtained. This is intuitively correct. The simulation results show that the proposed scheme achieves a higher resource efficiency than other existing schemes in $[1,5]$.

The curves in Fig. 4 show the performance analysis in terms of the system fairness. In this study, system fairness is estimated how to distribute the resource fairly among BSs. To characterize this fairness notion, we follow the Jain's fairness index $\left(F_{\text {index }}\right)$ [25], which has been frequently used to measure the fairness of network resource allocations.

$$
F_{\text {index }}=\left(\sum_{i=1}^{n} \mathscr{R}_{i}\right)^{2} /\left(n \times \sum_{i=1}^{n}\left(\mathscr{R}_{i}\right)^{2}\right)
$$

The range of $F_{\text {index }}$ is varied from 0 to 1 . On the basis of NBS and KSBS bargaining solutions, the proposed scheme re-distributes fairly the resource to BSs. Therefore, the proposed scheme can maintain an excellent system fairness than other existing schemes.

Figure 5 presents the performance comparison in terms of the QoS satisfaction probability. In this study,

Table 1 Application and system parameters used in the simulation experiment

\begin{tabular}{|c|c|c|c|c|}
\hline Application type & Applications & Cloud resource type & Minimum resource requirement & Maximum resource requirement \\
\hline I & Voice telephony & CPU & $30 \mathrm{MHz}$ & $60 \mathrm{MHz}$ \\
\hline$\|$ & Video-phone & CPU & $60 \mathrm{MHz}$ & $120 \mathrm{MHz}$ \\
\hline III & Remote-login & CPU & $15 \mathrm{MHz}$ & $40 \mathrm{MHz}$ \\
\hline IV & Tele-conference & CPU & $60 \mathrm{MHz}$ & $150 \mathrm{MHz}$ \\
\hline Parameter & Value & \multicolumn{3}{|l|}{ Description } \\
\hline$n$ & 5 & \multicolumn{3}{|l|}{ the number of $B S s$} \\
\hline$T_{Q}$ & $5 \mathrm{GHz}$ & \multicolumn{3}{|c|}{ Total cloud capacity (CPU computation capacity) } \\
\hline$p_{\min ,} p_{\max }$ & $0.1,1$ & \multicolumn{3}{|c|}{ The pre-defined minimum and maximum price boundaries } \\
\hline$d_{i, 1} \leq i \leq n$ & 0 & \multicolumn{3}{|c|}{ A payoff at the time of disagreement } \\
\hline Parameter & Initial & \multicolumn{2}{|l|}{ Description } & Values \\
\hline$p$ & 0.5 & \multicolumn{2}{|c|}{ The unit price for cloud resource } & Dynamically adjustable \\
\hline
\end{tabular}




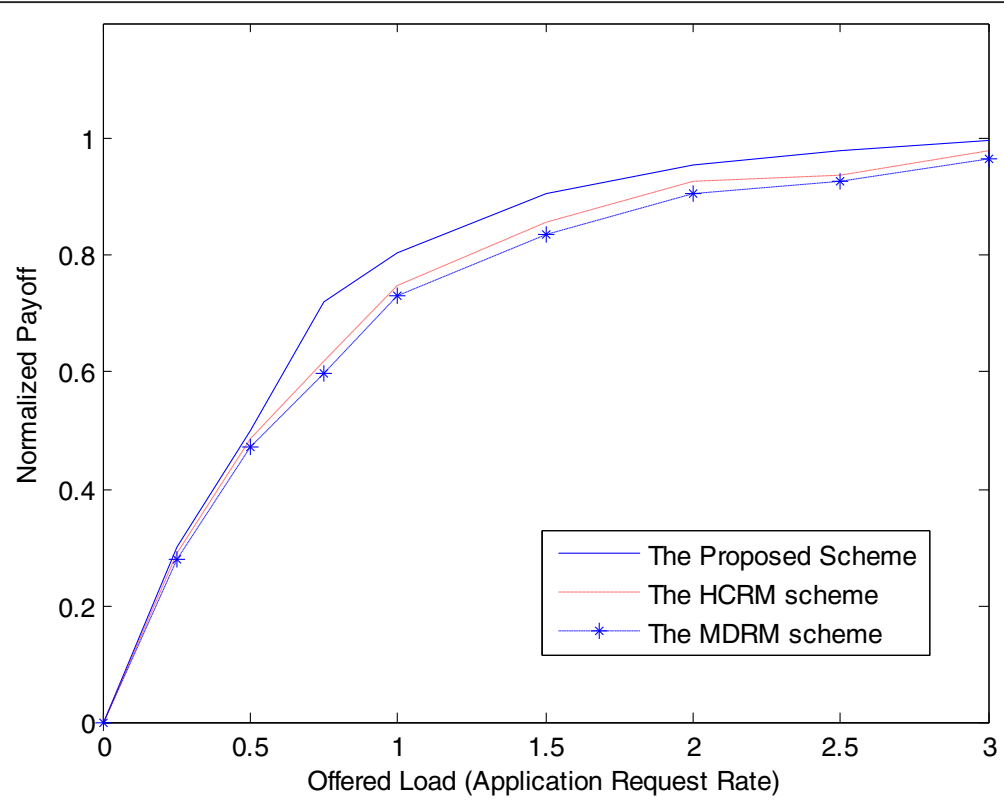

Fig. 2 Higher normalized payoff compared to the HCRM and MDRM schemes

QoS satisfaction probability is estimated as the rate of the successfully completed service applications. As the application requests increase, the average amount of available resource decreases. Thus, application services are likely to be closed incompletely; QoS satisfaction probability decreases. To improve the QoS satisfaction probability, our game-based approach iteratively adjusts the allocated resource in a step-by-step manner. The simulation results show that the proposed scheme achieves a higher QoS satisfaction probability than other existing schemes.

From the simulation results in Figs. 2, 3, 4, and 5, it can be seen that the performance trends of all the schemes are very similar. This is because the main design goals of all the schemes are the same. However, by employing two-stage news-vendor game approach, our proposed scheme adaptively responds to the current $\mathrm{C}$ RAN system conditions and efficiently solves the



Fig. 3 Higher resource efficiency compared to the HCRM and MDRM schemes 


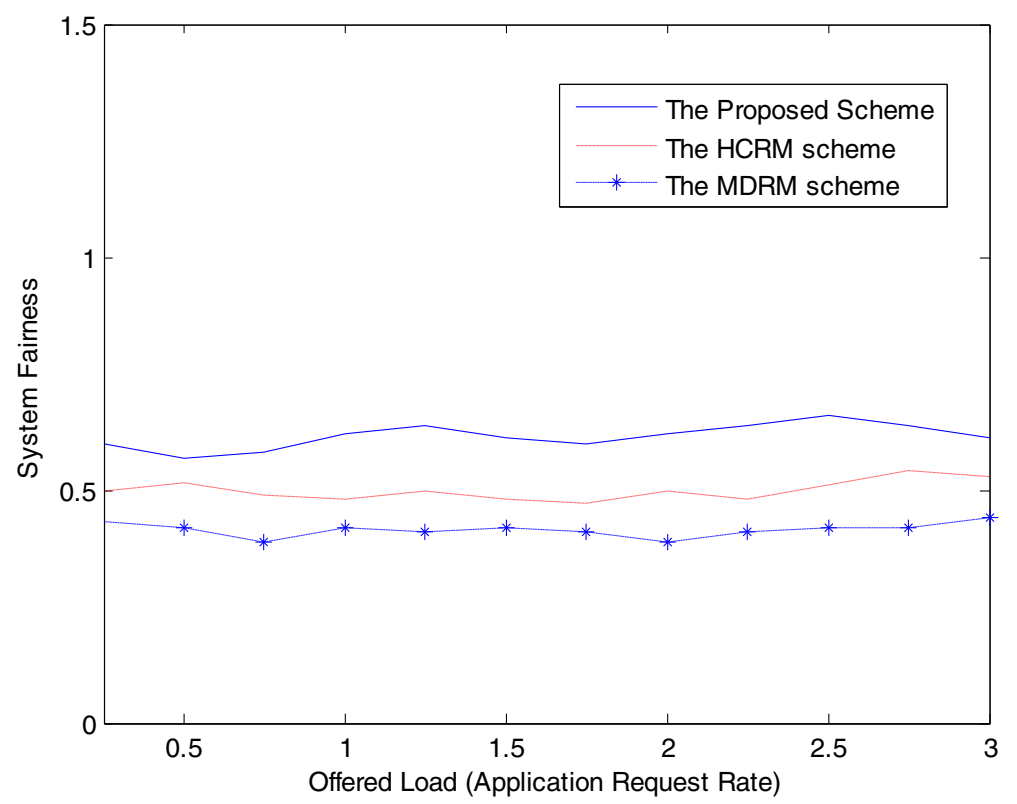

Fig. 4 Higher system fairness compared to the HCRM and MDRM schemes

resource allocation problems. Therefore, we can say that our proposed scheme is flexible, adaptable, and able to sense the dynamic changing C-RAN network environment; it is essential in order to be close to the optimized system performance while ensuring a better resource efficiency and QoS provisioning.

\section{Conclusions}

As a new model of distributed computing, all kinds of distributed resources are virtualized to establish a shared resource pool through C-RAN systems. C-RAN solution enables dynamic on-demand response, combining collaborative radio and real-time cloud infrastructure while providing convenient and configurable resources. Therefore, dynamic and efficient mechanism for rapidly scaling cloud resources is becoming a hot spot in research areas. In this study, we propose a novel resource allocation scheme based on the news-vendor game model. The main goal of our proposed scheme is to maximize system performance while ensuring service QoS. To

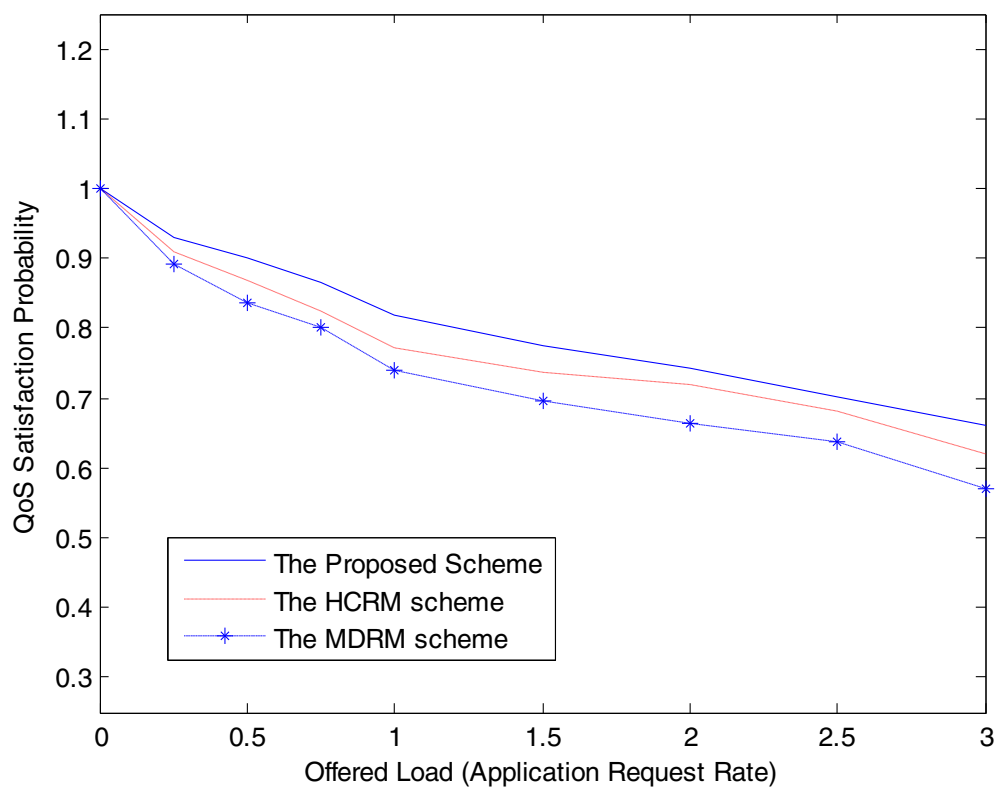

Fig. 5 Higher QoS satisfaction probability compared to the HCRM and MDRM schemes 
satisfy this goal, we develop a two-stage game mechanism. The important feature of our proposed scheme is its adaptability, flexibility, and responsiveness to current C-RAN conditions. The simulation results show that the proposed scheme can maintain an excellent C-RAN system performance as compared with the other existing schemes. For future research, we wish to extend the concept presented here in combination with security issues. Our game-based approach can be generalized to model various defense mechanisms and can be extended to address different security provisioning problems in dynamic C-RAN environments.

\section{Acknowledgements}

This research was supported by the MSIP (Ministry of Science, ICT and Future Planning), Korea, under the ITRC (Information Technology Research Center) support program (IITP-2016-H8501-16-1018) supervised by the IITP (Institute for Information \& Communications Technology Promotion) and was supported by Basic Science Research Program through the National Research Foundation of Korea (NRF) funded by the Ministry of Education (NRF-2015R1D1A1A01060835).

\section{Author' contributions}

Sungwook Kim is a sole author of this work and ES (i.e., participated in the design of the study and performed the statistical analysis).

\section{Competing interests}

The author declares that he has no competing interests.

Received: 12 January 2016 Accepted: 29 June 2016

Published online: 04 July 2016

\section{References}

1. B Addis, D Ardagna, B Panicucci, MS Squillante, L Zhang, A hierarchical approach for the resource management of very large cloud platforms. IEEE Trans. Dependable Secure Comput. 10(5), 253-272 (2013)

2. $Z$ Htike, CS Hong, S Lee, The life cycle of the rendezvous problem of cognitive radio ad hoc networks: a survey. JCSE 7(2), 81-88 (2013)

3. W Zhu, C Lee, A new approach to web data mining based on cloud computing. JCSE 8(4), 181-186 (2014)

4. K Sungwook, Dynamic C-RAN resource sharing scheme based on a hierarchical game approach. EURASIP J. Wirel. Commun. Netw. 2016(3), 1-12 (2016)

5. S Vakilinia, D Qiu, MM Ali, Optimal multi-dimensional dynamic resource allocation in mobile cloud computing. EURASIP J. Wirel. Commun. Netw. 2014(201), 1-14 (2014)

6. K Sungwook, Game theory applications in network design (IGI Global, Hershey, PA., U.S.A 2014)

7. WJ Stevenson, Operations management, 10th edition (McGraw-Hill, Columbus, OH, U.S.A 2009)

8. B Malakooti, Operations and production systems with multiple objectives (John Wiley \& Sons, Hoboken, NJ. U.S.A 2014)

9. KA Arrow, TE Harris, J Marschak, Optimal inventory policy. Econometrica, no. 19, 1951, pp. 250-1951

10. Y Zhiyuan, FR Yu, B Shengrong, Joint cloud computing and wireless networks operations: a game theoretic approach. IEEE GLOBECOM, 2014, pp. 4977-4982

11. D Niyato, AV Vasilakos, K Zhu, Resource and revenue sharing with coalition formation of cloud providers: game theoretic approach. IEEE/ACM CCGrid'2011, 2011, pp. 215-224

12. Z Ali, RU Rasool, P Bloodsworth, Social networking for sharing cloud resources. IEEE Cloud and Green Computing, 2012, pp. 160-166

13. S Caton, C Haas, K Chard, K Bubendorfer, OF Rana, A social compute cloud: allocating and sharing infrastructure resources via social networks. IEEE Trans. Serv. Comput. 7(3), 359-372 (2014)

14. Y Wang, C Guo, T Li, Q Xu, Secure two-party computation in social cloud based on reputation. IEEE $9^{\text {th }}$ WAINA, 2015, pp. 242-245
15. W Zhong, Y Fang, S Jin, K-K Wong, S Zhong, Z Qian, Joint Resource Allocation for device-to-device communications underlaying uplink MIMO cellular networks. IEEE J. Sel. Areas Commun. 33(1), 41-54 (2015)

16. W Zhong, G Chen, S Jin, K-K Wong, Relay selection and discrete power control for cognitive relay networks via potential game. IEEE Trans. Signal Process. 62(20), 5411-5424 (2014)

17. A Checko, HL Christiansen, Y Yan, L Scolari, G Kardaras, MS Berger, L Dittmann, Cloud RAN for mobile networks - a technology overview. IEEE Commun. Surv. Tutorials 17(1), 405-426 (2015)

18. W Jianning, No-centralize newsvendor model with re-distributed decisionmaking power. IEEE IITA'2009, 2009, pp. 237-240

19. B Xie, W Zhou, C Hao, X Ai, J Song, A novel bargaining based relay selection and power allocation scheme for distributed cooperative communication networks. IEEE Vehicular Technology Conference (VTC 2010), 2010, pp. 1-5

20. Z Yu, Z Hongtu, Study on negotiation strategy. International Conference On Power System Technology 2002, 2002, pp. 1335-1338

21. P Miao, F Yuguang, Bargaining based pairwise cooperative spectrum sensing for cognitive radio networks. IEEE MILCOM 2008, 2008, pp. 1-7

22. Y Park, S Kim, Bargaining based smart grid pricing model demand side scheduling management. ETRI J. 37(1), 197-202 (2015)

23. K Sungwook, Dynamic online bandwidth adjustment scheme based on Kalai-Smorodinsky bargaining solution. IEICE Trans. Commun. E93.B(7), 1935-1938 (2010)

24. E Zehavi, A Leshem, Alternative bargaining solutions for the interference channel. IEEE CAMSAP'2009, 2009, pp. 9-12

25. K Sungwook, Stackelberg game-based power control scheme for efficiency and fairness tradeoff. IEICE Trans. Commun. E94.B(8), 2427-2430 (2011)

\section{Submit your manuscript to a SpringerOpen ${ }^{\circ}$ journal and benefit from:}

- Convenient online submission

- Rigorous peer review

- Immediate publication on acceptance

- Open access: articles freely available online

- High visibility within the field

- Retaining the copyright to your article

Submit your next manuscript at $>$ springeropen.com 